\title{
Electromagnetically Interfered Energy Metering Resulting from Droop of Current Transducers
}

\author{
Bas ten Have \\ University of Twente \\ Enschede, The Netherlands \\ email: bas.tenhave@utwente.nl
}

\author{
Niek Moonen \\ University of Twente \\ Enschede, The Netherlands
}

\author{
Frank Leferink \\ University of Twente, Enschede \\ THALES Nederland B.V., Hengelo \\ The Netherlands
}

\begin{abstract}
Non-linear equipment is increasingly being used in household situations to increase the efficiency of the power consumption of equipment. This results in conducted electromagnetic interference problems due to the switching of the equipment, which draws pulsed currents with high peak values and fast rising slopes. Accordingly, static energy meters, used to measure the energy consumption in households, show misreadings in conjunction with such pulsed currents. Therefore, a control meter is under construction which is used to validate the readings of an installed static energy meter. When validating the control meter, energy metering errors were found resulting from current droop of the current transducers. The droop in the current response multiplied with the voltage resulted in large energy metering errors of $38 \%$. While no issues where found for linear, resistive, loads. This shows the need for pulsed immunity tests to validate energy measurement systems, because linear tests have not shown to be problematic.
\end{abstract}

Index Terms-Current droop, electromagnetic interference, non-linear, pulsed currents, static energy meter.

\section{INTRODUCTION}

Traditional equipment used by consumers in household situations consisted of linear equipment, where the current is sinusoidal and there might be a phase shift with the voltage. However, nowadays the use of non-linear equipment has increased, in order to increase the efficiency of the equipment by reducing its power consumption. This results in many conducted electromagnetic interference (EMI) problems in the frequency range from dc to $150 \mathrm{kHz}$ [1], [2], caused by the switching of such equipment which draws pulsed currents with high peak values, and thus a high $\mathrm{d} V / \mathrm{d} t$ and $\mathrm{d} I / \mathrm{d} t$. Such pulsed currents are causing errors of static energy meter readings, and errors are found due to dimmed lighting equipment utilizing light emitting diode (LED) and compact fluorescent lighting (CFL) technology [3], [4], and a speed controlled water pump [5], [6]. Maximum experimental errors of $2675 \%$ are found [7]. This is a meaningful problem, as static energy meters are rolled out in the European Union to measure the energy consumption in household situations. These reported errors might result in under or over billing of consumers.

For consumers confidence it is of importance to validate the accuracy of the static energy meter that is installed at their residence. Therefore, a control meter is under construction

This project 17NRM02 MeterEMI has received funding from the EMPIR programme co-financed by the Participating States and from the European Union's Horizon 2020 research and innovation programme. to check the installed static energy meter. It should be made portable for an easy installation in an on-site test scenario, therefore analyzers for lab purposes are too bulky. Hence, the use of a compact commercial off-the-shelf (COTS) power quality analyzer is evidenced. Such analyzer could also be equipped with an interface to monitor the installed static energy meter based on [8]. To test the suitability of such a device it is validated, i.e. can it accurately measure the currents from a water pump that interfered with static energy meter readings in previous research [5]. However, incorrect energy readings of the power quality analyzer were found during these validation measurements.

The purpose of this paper is to analyze the incorrect energy readings of the COTS power quality analyzer and to find the root-cause. This is done by the analysis of the measured current response of the current transducer (CT) that is used along with the power quality analyzer. Also different CT models are tested, which are non-invasive and use either the current transformer or Rogowski principle for measuring the current. A current droop in the response of the CTs was found and is resulting from the magnetization current in a current transformer [9]-[11] and in case of a Rogowski coil from the integrator [12]. Due to this current droop errors in the energy measurement occurred. It was evidenced that linear, resistive, loads could be measured correctly. Therefore, testing the immunity of energy measurements using frequencydomain tests as is done in the IEC 61000-4-19 standard [13] is not sufficient to determine the immunity against non-linear, pulsed, currents as was already evidenced in [14].

The rest of this paper is organized as follows: In Section II the control meter built using a COTS power quality analyzer is introduced briefly. After which Section III describes the validation measurements and results. Then, Section IV follows by a root-cause analysis of the incorrect readings obtained in the validation measurements. Next, those results are discussed in Section V. And finally, Section VI concludes this work.

\section{Control meter to VAlidate installed Static ENERGY METERS}

A control meter able to validate static energy meters installed at a consumers' residence is under construction. A COTS power quality analyzer model PQube 2 manufactured by Power Standards Lab is used. The device can log the energy, 
power, currents and voltages, which are also measured by the installed static energy meter. Furthermore, we added an interface to readout the installed static energy meter based on the LED pulse counter of the meter according to [8]. Additionally, triggers are used to capture detailed waveform data of basic low-frequency conducted phenomena in the voltage grid, such as dips, surges, fluctuations, harmonic distortion, transients, and unbalance. The voltage measurement channel is connected directly to the grid to measure the fundamental voltage. Non-invasive CTs are connected to measure the currents safely without making any adjustments to the system. Hence, CTs model SCS-075-0050A:0.333V manufactured by Power Standards Lab are used to measure the current. The schematic of the working principle and photo of the described reference device are depicted in Fig. 1 and Fig. 2, respectively.

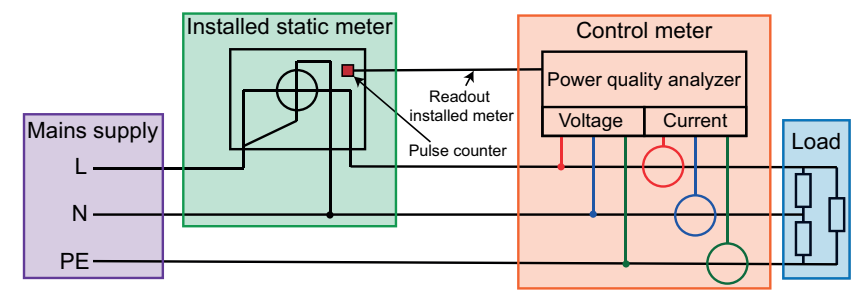

Fig. 1. Schematic of the working principle of the control meter in an one-phase system.

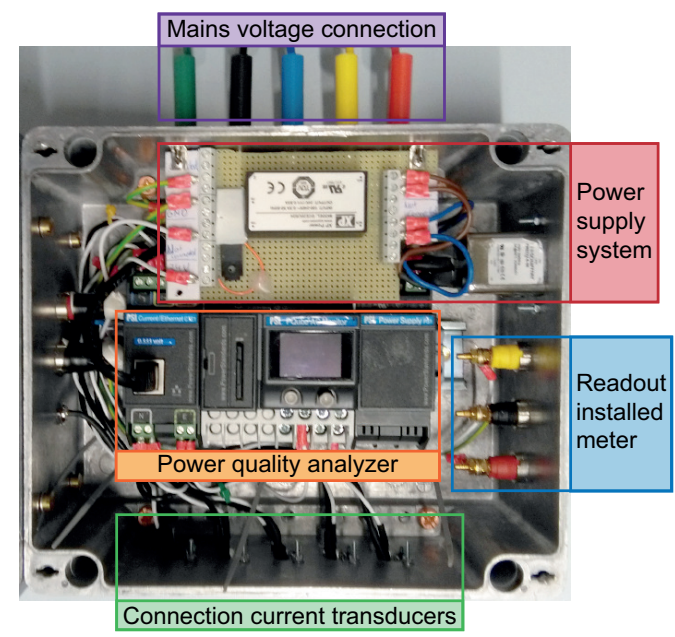

Fig. 2. Photograph of the control meter.

\section{VALIDATION OF THE CONTROL METER}

The aim here is to find if the control meter provides an accurate reference for installed static energy meters. Therefore, it is tested if the device is capable of measuring signals known to result in static energy meter errors in previous research [5], as it should be immune to such interference in order to provide an accurate reference for installed static energy meters. Further, the device is also tested against a chopped sinusoidal load.

\section{A. Validation method}

The control meter is connected in such a way to measure the energy consumption of a load. Seven different load situations are tested, which cover three linear loads and four nonlinear loads. These represent standard (linear) test signals of different amplitudes and non-linear signals known to cause errors in static energy meter readings, respectively. Linear signals of 190,800 , and $1800 \mathrm{~W}$ (R190, R800, and R1800, respectively), that are generated using a heater, are used. And non-linear signals are used that are generated by a water pump at dimming level 1, 4, and 9 (WP1, WP4, and WP9, respectively), and using a resistive signal of $190 \mathrm{~W}$ which is dimmed (chopped) using a phase firing dimmer at 145 degrees (R190D145). The characteristics of these test signals are depicted in Table I. As a reference instrument a power analyzer model WT500 manufactured by Yokogawa is used. It has a basic power accuracy of $0.1 \%$, high sampling rate and experience has shown that we can trust the readings for reference as we are focused on EMI, and not on metrology. The schematic of the validation setup is shown in Fig. 3. Then, the deviation in measured energy by the control meter is compared to the reference instrument using,

$$
\text { Deviation }(\%)=\frac{E_{\text {dut }}-E_{\text {ref }}}{E_{\text {ref }}} \cdot 100 \%
$$

where $E_{\text {dut }}$ is the measured energy of the device under test, which is the control meter, and $E_{\text {ref }}$ is the energy measured by the reference instrument. This method is similar to the method used to test the readings of static energy meters in [5].

TABLE I. Time-domain parameters of the test signals.

\begin{tabular}{l|r|r|r|r|r}
\hline \hline Test signal & $\begin{array}{r}\text { Charge } \\
(\mathbf{m C})\end{array}$ & $\begin{array}{r}\text { Crest } \\
\text { factor }\end{array}$ & $\begin{array}{r}\text { Peak } \\
\text { value }(\mathbf{A})\end{array}$ & $\begin{array}{r}\text { Pulse } \\
\text { width }(\mathbf{m s})\end{array}$ & $\begin{array}{r}\text { Slope } \\
(\mathbf{A} / \boldsymbol{\mu s})\end{array}$ \\
\hline \hline R190 & 16.9 & 1.4 & 1.3 & 20 & $3.5 \cdot 10^{-4}$ \\
\hline R800 & 66.4 & 1.4 & 5.2 & 20 & $1.3 \cdot 10^{-3}$ \\
\hline R1800 & 149.3 & 1.4 & 11.7 & 20 & $3.0 \cdot 10^{-3}$ \\
\hline WP1 & 4.8 & 9.1 & 17.2 & 0.4 & 2.8 \\
\hline WP4 & 6.6 & 8.5 & 17.2 & 0.8 & 2.2 \\
\hline WP9 & 7.1 & 8.0 & 17.1 & 0.7 & 2.0 \\
\hline R190D145 & 0.3 & 9.9 & 0.8 & 1.2 & 0.3 \\
\hline \hline
\end{tabular}

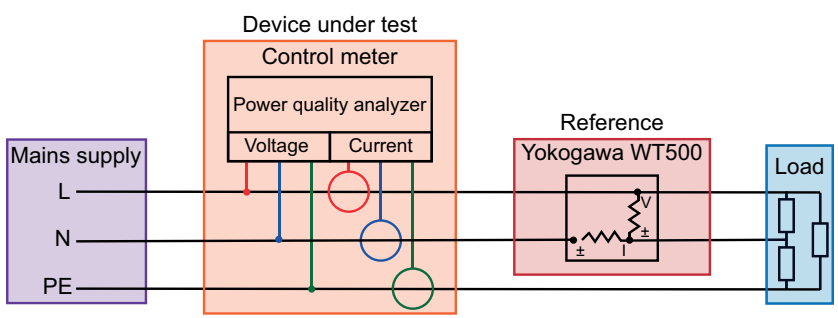

Fig. 3. Schematic of the validation method to test the energy reading of the control meter.

\section{B. Validation results}

The validation results are summarized in Table II. Here, in red the deviations exceeding the maximum permissible limits for electricity meters in Europe with approval of the 
European Measuring Instrument Directive (MID) according to the European standard EN 50470-3:2006 [15], which is 2.5\% for Class A electricity meters, are highlighted. The results show that for WP1 and WP4 deviations above the permissible limits occur, while the other test signals cause no deviations exceeding the permissible limits.

TABLE II. Validation results, showing the energy deviations of the control meter.

\begin{tabular}{l|r|r|r}
\hline \hline Test signal & $\boldsymbol{E}_{\text {dut }}(\mathbf{W h})$ & $\boldsymbol{E}_{\boldsymbol{r e f}}(\mathbf{W h})$ & Deviations (\%) \\
\hline \hline R190 & 3.4 & 3.5 & -2 \\
\hline R800 & 21.7 & 22.9 & -2 \\
\hline R1800 & 27.1 & 27.4 & -1 \\
\hline WP1 & 2.9 & 2.1 & 38 \\
\hline WP4 & 3.0 & 2.5 & 18 \\
\hline WP9 & 4.3 & 4.4 & -1 \\
\hline R190D145 & 14.1 & 14.3 & -2 \\
\hline \hline
\end{tabular}

\section{ROOT-CAUSE ANALYSIS}

In order to find the root-cause of the deviations in the energy readings of the control meter, the most critical situation is analyzed in more detail, i.e. when it is loaded with WP1. A zoomed version of the response of the current transducer is visible in Fig. 4(a) and Fig. 4(b) shows one period of the complete wave. It is clear that in the time period in which no pulse is present, and the signal should have a zero value, a sawtooth in the zoomed response is present. The presence of this saw-tooth is likely to produce the observed energy deviation.

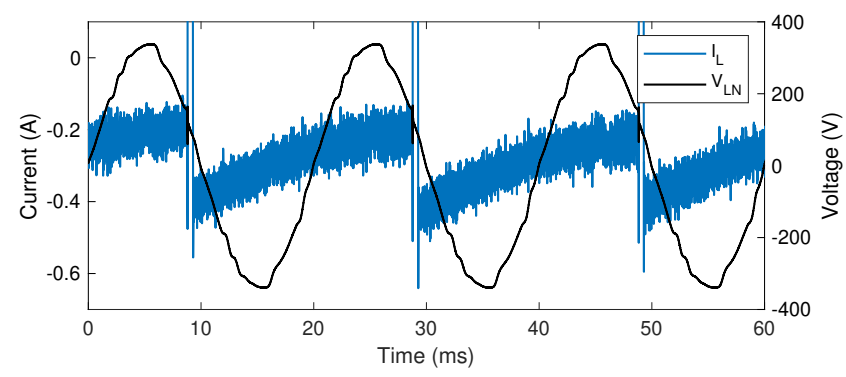

(a) Zoomed response

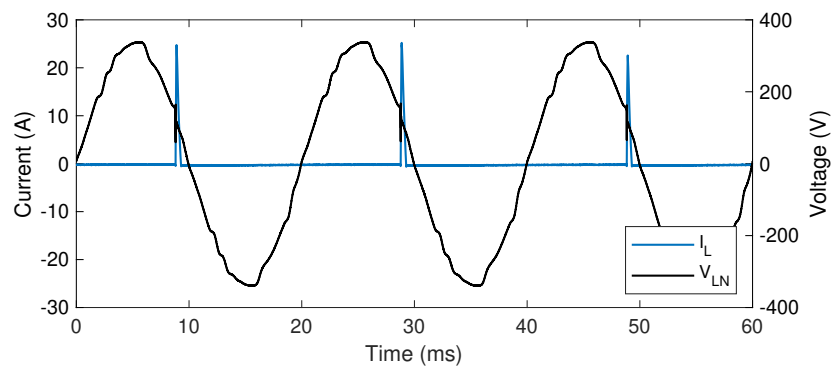

(b) Complete response

Fig. 4. Response of CT1 when loaded with WP1.

Because the reason of this saw-tooth is unclear and might be due to the used CT, additional measurements are performed when the system is loaded with WP1, but using different CTs. Hence, two extra models are tested, namely: model CWT3 from Power Electronic Measurements (CT2), and model TA189 from Pico Technology (CT3). These are used because CT2 is a Rogowski coil and provides a different measurement technique, and CT3 was already proven to give accurate responses with pulsed signals in [16]. The initially used CT is also used, and is referred to as CT1 in what follows. A photograph of these CTs is depicted in Fig. 5. The voltage is measured using a differential voltage probe model TA043 from Pico Technology. The responses of the CTs and the voltage are measured synchronously using a Picoscope 5444D pc-based oscilloscope. The average power over 10 cycles resulting from the product between the voltage and current is determined, and is compared to the same reference as used before. Using a fast Fourier transform (FFT) the power over frequency is determined and then accumulated over the frequency ranges of interest in Table III. The schematic of this experiment is depicted in Fig. 6.

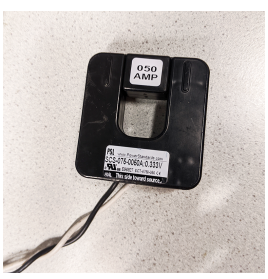

(a) $\mathrm{CT} 1$

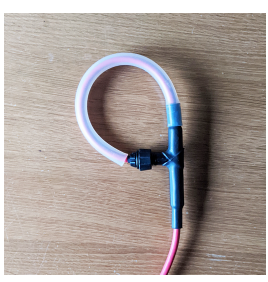

(b) CT2

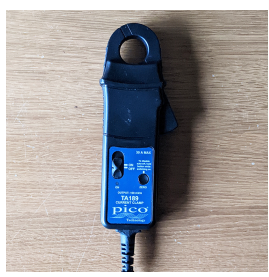

(c) $\mathrm{CT} 3$
Fig. 5. Photograph of the used CTs.

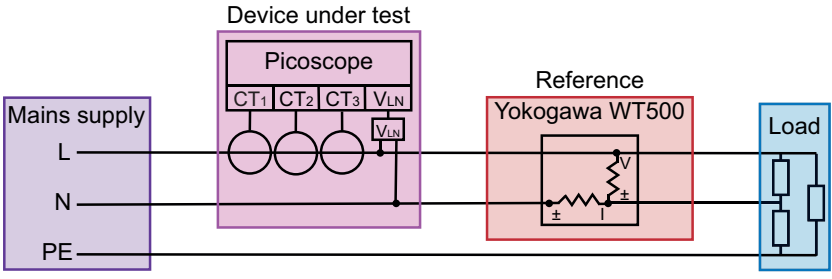

Fig. 6. Schematic of the measurements with three different CTs.

The results are presented in Fig. 7 and Table III. For both CT1 and CT2 the saw-tooth behavior is visible, and also power deviations of $31 \%$ and $35 \%$ are measured, respectively. From the power per frequency it is clear that the saw-tooth, which is repeating with the fundamental frequency of $50 \mathrm{~Hz}$, is resulting in a higher measured power at the fundamental, as this is the only frequency where differences between the CTs are observed.

TABLE III. Power readings per frequency.

\begin{tabular}{l|r|r|r}
\hline \hline Frequency (Hz) & CT1 & CT2 & CT3 \\
\hline \hline$<50$ & $0.0 \mathrm{~W}$ & $0.0 \mathrm{~W}$ & $0.0 \mathrm{~W}$ \\
\hline 50 & $41.3 \mathrm{~W}$ & $42.5 \mathrm{~W}$ & $31.9 \mathrm{~W}$ \\
\hline$>50-1000$ & $0.8 \mathrm{~W}$ & $0.7 \mathrm{~W}$ & $0.7 \mathrm{~W}$ \\
\hline$>1000-10000$ & $-2.0 \mathrm{~W}$ & $-2.0 \mathrm{~W}$ & $-2.0 \mathrm{~W}$ \\
\hline$>10000$ & $0.0 \mathrm{~W}$ & $0.0 \mathrm{~W}$ & $0.0 \mathrm{~W}$ \\
\hline Total & $\mathbf{4 0 . 1} \mathrm{W}$ & $\mathbf{4 1 . 2 ~ W}$ & $\mathbf{3 0 . 6} \mathrm{W}$ \\
\hline Deviation & $31 \%$ & $35 \%$ & $0 \%$ \\
\hline \hline
\end{tabular}

Next, it is verified whether the measured pulsed part is similar and the difference between the responses of the different 


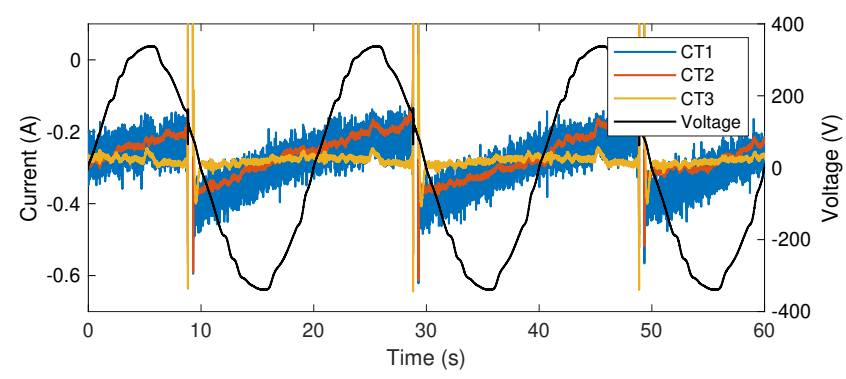

(a) Zoomed response

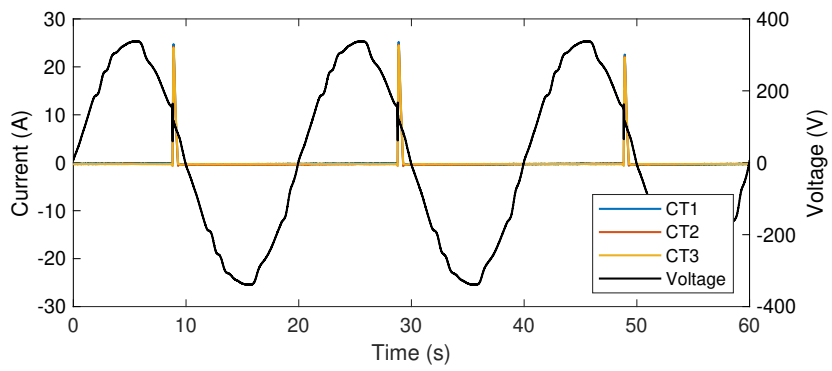

(b) Complete response

Fig. 7. Response of the CTs measuring load WP1.

TABLE IV. Time-domain parameters of responses CTs.

\begin{tabular}{l|r|r|r}
\hline \hline Parameter & CT1 & CT2 & CT3 \\
\hline \hline Charge $(\mathrm{mC})$ & 5.6 & 5.4 & 5.5 \\
\hline Crest factor & 10.8 & 10.8 & 10.7 \\
\hline Peak value $(\mathrm{A})$ & 23.5 & 23.0 & 22.8 \\
\hline Pulse duration $(\mathrm{ms})$ & $4.8 \cdot 10^{-1}$ & $4.6 \cdot 10^{-1}$ & $4.9 \cdot 10^{-1}$ \\
\hline Slope $(\mathrm{A} / \mu \mathrm{s})$ & 1.2 & 1.6 & 1.7 \\
\hline \hline
\end{tabular}

CTs is due to the existence of the saw-tooth only. Therefore, the pulses are extracted from the original measured response and the time-domain parameters are determined using the parametric waveform model presented in [17]. The resulting parameters are presented in Table IV. Here, it is shown that all parameters are similar and the difference between the measured powers originates most likely due to the saw-tooth behavior of CT1 and CT2. The product of the saw-tooth and the voltage results in an additional power of approximately $10 \mathrm{~W}$.

\section{DISCUSSION}

In this paper a situation is shown where a pulsed current from a commercial water pump interfered with the response of two different current transducers resulting in energy misreadings of a power quality analyzer. These two current transducers used either the current transformer or Rogowski coil principle to measure the current. And the CTs response shows a discrepantcy which was a saw-tooth at the fundamental frequency, this behavior is also called droop and occurs due to the behavior of the $\mathrm{CT}$ at dc.

To produce a flux in a current transformers' core for a finite permeability, a supporting current is needed, called the magnetization current [9]-[11]. This could be modelled in an equivalent circuit by a magnetisation inductance $\left(Z_{\mathrm{E}}\right)$, also known as open circuit inductance, Fig. 8. It is determined by the dimensions and permeability of the core material [11]. This inductance is ideally infinite (i.e. infinite permeability), and when the core saturates it tends to zero [9]. The current transformer only induces a current in the secondary winding for a changing current in the primary winding. When the current is constant, as is the case for the bottom and top part of a pulsed current, no current is induced in the secondary and the current level will drop exponential with a time constant determined by $Z_{\mathrm{E}}, R_{\mathrm{S}}$, and $Z_{\mathrm{s}}$. This behavior is called droop and results in the observed saw-tooth in the current response.

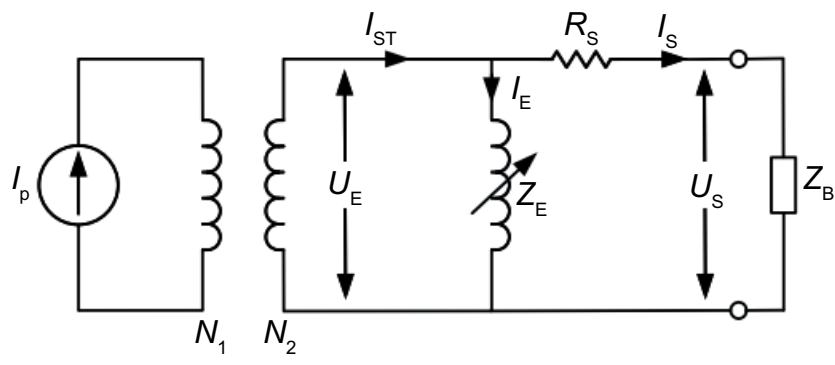

Fig. 8. Equivalent circuit of a current transformer [10].

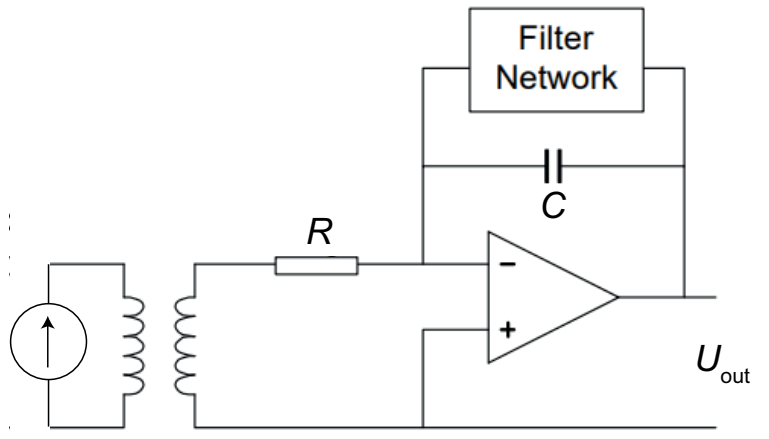

Fig. 9. Equivalent circuit of a Rogowski coil with an active integrator.

Likewise, for a Rogowski coil the droop occurs due to the the capacitor in the integrator, where the equivalent circuit of a Rogowski coil with an active integrator is depicted in Fig. 9. Again for a constant current there is no current induced at the secondary winding, which results in an exponential decay which is affected with the time constant determined by $R$ and $C$ [12].

As a result, when CTs are used to measure pulsed currents discrepancies in the current response occur. Where the response slightly varies when no pulse and thus current is present in the measured signal, and an additional saw-tooth is present in the response. The product of the voltage and the saw-tooth in the current response, results in a measured energy additional to the energy of the pulse. Which leads to the occurrence of energy errors outside the permissible limits for electricity meters in Europe according to the European standard EN50470-3:2006 [15]. While for linear sinusoidal currents as used in immunity tests, no issues for the energy 
measurement system are observed. This shows the need of immunity tests using pulsed currents to test the limits of energy metering systems, and thus signals with a combination of frequencies. Instead of using frequency-domain tests as is done in the IEC 61000-4-19 standard [13]. Because pulsed test methods will indicate the errors in the energy measurement due to the current droop, while single-tone currents do not indicate this problem. The need for pulsed current test methods was already pointed out in [14]. Likewise, it is worthwhile to look into time-domain characterization methods for current transducers that use pulsed currents, as was already proposed in [16].

\section{CONCLUSION}

Erroneous energy measurements are shown to exists as a result of a discrepant response of two CTs used in conjunction with a power quality analyzer. The discrepant response was caused by current droop of the CTs when measuring pulsed currents from a water pump. This caused an additional sawtooth in the measured current, which resulted in an extra, erroneous, energy consumption. While it was also shown that for linear loads no issues for the energy measurement system occurred. This shows the need to use pulsed currents to test the limits of an energy metering system in immunity tests, because traditional frequency-domain tests will not indicate errors that are caused by the current droop of CTs.

\section{REFERENCES}

[1] K. Murakawa, N. Hirasawa, H. Ito, and Y. Ogura, "Electromagnetic interference examples of telecommunications system in the frequency range from $2 \mathrm{kHz}$ to $150 \mathrm{kHz}$," in 2014 International Symposium on Electromagnetic Compatibility, Tokyo, Japan, 2014, pp. 581-584.

[2] F. Leferink, "Conducted interference, challenges and interference cases," IEEE Electromagnetic Compatibility Magazine, vol. 4, no. 1, pp. 78-85, Apr. 2015.

[3] F. Leferink, C. Keyer, and A. Melentjev, "Static energy meter errors caused by conducted electromagnetic interference," IEEE Electromagnetic Compatibility Magazine, vol. 5, no. 4, pp. 49-55, 2016.

[4] Z. Marais, H. E. Van den Brom, G. Rietveld, R. Van Leeuwen, D. Hoogenboom, and J. Rens, "Sensitivity of static energy meter reading errors to changes in non-sinusoidal load conditions," in 2019 International Symposium on Electromagnetic Compatibility (EMC Europe 2019), Barcelona, Spain, 2019, pp. 202-207.
[5] B. ten Have, T. Hartman, N. Moonen, C. Keyer, and F. Leferink, "Faulty Readings of Static Energy Meters Caused by Conducted Electromagnetic Interference from a Water Pump," in Renewable Energy and Power Quality Journal (RE\&PQJ), Santa Cruz de Tenerife, Spain, 2019, pp. 15-19.

[6] B. ten Have, T. Hartman, N. Moonen, and F. Leferink, "Misreadings of Static Energy Meters due to Conducted EMI caused by Fast Changing Current," in 2019 Joint International Symposium on Electromagnetic Compatibility and Asia-Pacific International Symposium on Electromagnetic Compatibility, Sapporo, Japan, 2019, pp. 445-448.

[7] B. ten Have, T. Hartman, N. Moonen, and F. Leferink, "Inclination of Fast Changing Currents Effect the Readings of Static Energy Meters," in 2019 International Symposium on Electromagnetic Compatibility (EMC Europe 2019), Barcelona, Spain, 2019, pp. 208-213.

[8] B. ten Have, C. Keyer, and F. Leferink, "Monitoring of Power Measured by Static Energy Meters for Observing EMI Issues," in 2018 International Symposium on Electromagnetic Compatibility (EMC Europe), Amsterdam, The Netherlands, 2018, pp. 903-907.

[9] S. Hodder, B. Kasztenny, N. Fischer, and Y. Xia, "Low SecondHarmonic Content in Transformer Inrush Currents - Analysis and Practical Solutions for Protection Security," in 2014 67th Annual Conference for Protective Relay Engineers, College Station, TX, USA, 2014, pp. 705-722.

[10] A. Hargrave, M. J. Thompson, and B. Heilman, "Beyond the Knee Point : A Practical Guide to CT Saturation," in 2018 71st Annual Conference for Protective Relay Engineers (CPRE), College Station, TX, USA, 2018, pp. 1-23.

[11] W. H. Portilla, "Analysis of the non-linear behaviour of the magnetisation inductance during the frequency response test of a transformer," IET Science, Measurement \& Technology, vol. 3, no. 8, pp. 1186-1193, Oct. 2019.

[12] W. F. Ray and C. R. Hewson, "Rogowski transducers for measuring large magnitude short duration pulses," in IEE Symposium on Pulsed Power 2000 (Digest No. 2000/053), London, United Kingdom, 2000, pp. $1-4$.

[13] Electromagnetic Compatibility (EMC) - Part 4-19: Testing and measurement techniques - Test for immunity to conducted, differential mode disturbances and signalling in the frequency range from $2 \mathrm{kHz}$ to 150 $\mathrm{kHz}$, at a.c. power port, IEC 61000-4-19 Std., 2012.

[14] B. ten Have, T. Hartman, N. Moonen, and F. Leferink, "Why Frequency Domain Tests Like IEC 61000-4-19 Are Not Valid; a Call for Time Domain Testing," in 2019 International Symposium on Electromagnetic Compatibility (EMC Europe 2019), Barcelona, Spain, 2019, pp. 124128.

[15] Electricity metering equipment (a.c.) - Part 3: Particular requirements - Static meters for active energy (class indexes A, B and C), EN 504703:2006 Std., 2006.

[16] B. ten Have, N. Moonen, and F. Leferink, "Time Domain Analysis of Current Transducer Responses using Impulsive Signals," IEEE Letters on Electromagnetic Compatibility Practice and Applications, vol. 3, no. 1, pp. 19-23, Mar. 2021.

[17] B. ten Have, M. A. Azpúrua, T. Hartman, M. Pous, N. Moonen, F. Silva, and F. Leferink, "Waveform Model to Characterize Time-Domain Pulses Resulting in EMI on Static Energy Meters," IEEE Transactions on Electromagnetic Compatibility (Early Access), pp. 1-8, Apr. 2021. 